\title{
$r$-PROCESS IN PROMPT SUPERNOVA EXPLOSIONS REVISITED
}

\author{
K. Sumiyoshi ${ }^{1}$, M. Terasawa ${ }^{2,3,4}$, G. J. Mathews ${ }^{3,5}$, T. Kajino ${ }^{2,3,6}$ S. Yamada $^{7}$, And \\ H. SUZUKI ${ }^{8}$ \\ sumi@la.numazu-ct.ac.jp
}

The Astrophysical Journal, Submitted 2001 March 23

\begin{abstract}
We reanalyze $r$-process nucleosynthesis in the neutron-rich ejecta from a prompt supernova explosion of a low-mass $\left(11 \mathrm{M}_{\odot}\right)$ progenitor. We have performed a general relativistic hydrodynamic simulation of adiabatic collapse and bounce using a relativistic nuclear-matter equation of state. The electron fraction $Y_{e}$ was fixed at a value such that the size of the inner collapsing core would be large enough to enable a prompt explosion to occur in the hydrodynamical calculation. Adopting the calculated trajectories of promptly ejected material, we explicitly computed the burst of neutronization due to electron captures on free protons in the photodissociated ejecta after the passage of the shock. The thermal and compositional evolution of the resulting neutron-rich ejecta originating from near the surface of proto-neutron star was obtained. These were used in nuclear reaction network calculations to evaluate the products of $r$-process nucleosynthesis. We find that, unlike earlier studies of nucleosynthesis in prompt supernovae, the amount of $r$-process material ejected per supernova is quite consistent with observed galactic $r$-process abundances. Furthermore, the computed $r$-process abundances are in good agreement with Solar abundances of $r$-process elements for $\mathrm{A}>100$. This suggests that prompt supernovae are still a viable $r$-process site. Such events may be responsible for the abundances of the heaviest $r$-process nuclei.
\end{abstract}

Subject headings: nuclear reactions, nucleosynthesis, abundances — stars: abundances — stars: neutron - supernovae: general

\footnotetext{
${ }^{1}$ Numazu College of Technology (NCT), Ooka 3600, Numazu, Shizuoka 410-8501, Japan

${ }^{2}$ Department of Astronomy, The University of Tokyo, Hongo 7-3-1, Bunkyo, Tokyo 113-0033, Japan

${ }^{3}$ National Astronomical Observatory, Osawa 2-21-1, Mitaka, Tokyo 181-8588, Japan

${ }^{4}$ Institute of Physical and Chemical Research (RIKEN) Hirosawa 2-1, Wako, Saitama 351-0198, Japan

${ }^{5}$ Center for Astrophysics, Department of Physics, University of Notre Dame, Notre Dame, Indiana 46556, USA

${ }^{6}$ The Graduate University for Advanced Studies, Osawa 2-21-1, Mitaka, Tokyo 181-8588, Japan

${ }^{7}$ Institute of Laser Engineering (ILE), Osaka University, Yamadaoka 2-6, Suita, Osaka 565-0871, Japan

${ }^{8}$ Department of Physics, Science University of Tokyo, Yamazaki 2641, Noda, Chiba 278-8510, Japan
} 


\section{Introduction}

The astrophysical site for the origin of elements by rapid neutron capture (the $r$-process) has been a mystery for some time. Numerous sites have been proposed as possible candidate environments (Hillebrandt 1978; Mathews \& Ward 1985; Mathews \& Cowan 1990; Meyer 1994). At the present time the most popular among proposed sites involves the neutrino-heated ejecta from a nascent neutron star (Woosley \& Hoffman 1992; Meyer et al. 1992; Woosley et al. 1994; Otsuki et al. 2000; Sumiyoshi et al. 2000; Terasawa et al. 2001). In Woosley et al. (1994) it was demonstrated that the Solar $r$-process abundances were well reproduced in material which has been ablated from the proto-neutron star in neutrino-driven winds.

There are, however, a few serious problems with this $r$-process paradigm. For one, elements with $A \sim 90$ are significantly overproduced by over a factor of $\sim 100$. Second, the requisite high entropy $(S / k \gtrsim$ $400)$ in the supernova simulations has not been duplicated by other independent theoretical studies (cf. Witti, Janka, \& Takahashi 1994; Takahashi, Witti, \& Janka 1994; Qian \& Woosley 1996). A third problem (Meyer, McLaughlin, \& Fuller 1998) is the possibility that neutrino-nucleus interactions could drastically alter the neutron to seed ratio rendering a satisfactory $r$-process more difficult.

On the other hand, other viable sites have been demonstrated to also account for the Solar $r$-process abundance pattern. For example, it has been shown (Freiburghaus, Rosswog, \& Thielemann 1999) that neutron-star mergers can produce the abundances of nuclei with $A \gtrsim 130$. Collapsing $\mathrm{O}-\mathrm{Ne}-\mathrm{Mg}$ cores resulting from progenitor stars of low mass $\sim 10 M_{\odot}$ also remain (Wheeler, Cowan, \& Hillebrandt 1998) as a promising site for the $r$-process. The present work, however, is primarily concerned with collapsing iron cores of $\sim 11 M_{\odot}$ progenitor stars.

In several early papers (e.g. Hillebrandt et al. 1976; Hillebrandt 1982; Hillebrandt et al. 1984) studies were made of $r$-process nucleosynthesis in the material hydrodynamically ejected during a prompt supernova explosion. In Hillebrandt et al. (1976) the $r$-process was thought to occur in the inner $\sim 0.43 \mathrm{M}_{\odot}$ of ejected material in which the neutron to proton ratio was taken to range from $n / p \sim 1$ to 7 . The problem with these earlier studies, however, is that it appeared that too much material was ejected to make a reasonable accounting for the galactic abundances of $r$-process nuclides. To account for the current galactic abundance of $r$-process material requires that only about $10^{-4} \mathrm{M}_{\odot}$ of $r$-process elements be ejected per supernova (cf. Mathews \& Cowan 1990; Woosley et al. 1994). This is much less than the $\left(\sim 0.4 \mathrm{M}_{\odot}\right)$ ejected in the Hillebrandt et al. (1976) model. Furthermore, it gradually became clear (Baron \& Cooperstein 1990, Bethe 1990, Suzuki 1994) that models of prompt supernovae could only be made to explode for a limited range of small core-masses, high lepton fractions, and equation of state parameters, and may not occur at all. Because of these dilemmas, prompt supernovae have fallen out of favor as an $r$-process paradigm in recent years.

However, recent studies (Heger et al. 2001) of progenitor cores based upon improved slower electron capture rates (Langanke \& Martinez-Pinedo 2000) have shown that smaller, cooler, iron cores with larger lepton fractions are a likely outcome of supernova progenitor-star evolution. This suggests the possibility that prompt explosions may indeed happen.

Given that a prompt explosion seems possible, the purpose of the present work is to revisit the question of whether prompt supernovae necessarily overproduce the abundances of $r$-process elements. Therefore, in the present work we perform new calculations of the $r$-process during a prompt supernova explosion. Our purpose is not to provide a fully detailed prompt explosion model, which will take some time to complete. Instead, our purpose here is merely to develop a basic prompt explosion model with enough content to examine the question of the implied $r$-process yield and relative abundances. Therefore, we begin with an adiabatic collapse simulation with the electron fractions $\left(Y_{e}\right)$ of the various zones fixed at the initialmodel values. This approximates the effect of the new diminished electron capture rates. This model produces a prompt explosion, both because the core bounce is energetic, and because the higher $Y_{e}$ leads to a small outer iron core which does not have enough material to dissipate the shock by photodissociation. It is adequate for our purpose.

A key part of the $r$-process calculation of the present work is that we perform post processing of the compositional change of the ejecta relevant to determining the amount of $r$-process material ejected. First, we consider the burst of neutronization due to electron capture on free protons after the shock heating and photodissociation of the ejecta. We show that 
the neutronization is rapid. Moreover, the amount of neutronized ejecta is much less than was assumed in earlier work. Consequently, the correct amount of neutron rich material is ejected to account for galactic abundances. Our network calculations of the ensuing $r$-process nucleosynthesis makes a reasonable reproduction of the Solar $r$-process abundance distribution for nuclei with $A \gtrsim 100$. This suggests that prompt supernovae remain as a viable candidate site for the $r$-process synthesis of heavy elements.

\section{Prompt Supernova Mechanism}

For some time it has been debated in the literature as to whether collapsing iron cores of supernova progenitors can explode via a prompt mechanism (Bethe 1990, Suzuki 1994). The question has been whether the core bounce itself can be sufficiently energetic to eject the outer layers of the star. It is always difficult to develop a supernova model which actually explodes (Bethe 1990, Suzuki 1994) and it has been clear for some time that if a prompt explosion is to occur it must involve a low-mass $\left(\sim 10-12 \mathrm{M}_{\odot}\right)$ progenitor star and a low-mass collapsing core (e.g. Baron \& Cooperstein 1990). Whether or not an explosion can occur depends upon a difference between two large numbers: the energy in the outward moving shock and the energy lost by the shock due to the photodissociation of nuclei. At present there are too many uncertainties in the input supernova physics to definitively exclude a prompt explosion mechanism for at least a limited range of core masses and equation of state parameters. Nevertheless, it is clear what ingredients are necessary. Two key factors which we invoke here to obtain a prompt explosion are a small total iron core mass and a large $Y_{e}$ as a result of slower electron capture rates. The important roles of these we now outline in a brief summary of the explosion mechanism

As the electron-degenerate iron core reaches its Chandrasekhar mass, the collapse divides into an inner homologously collapsing core and an outer core collapsing more slowly. A prompt explosion requires a small outer iron core so that the outward moving shock from the bounce of the inner core is not dissipated by photodissociation of the outer core. A small outer core can be achieved by minimizing the total Chandrasekhar mass of the iron core, while maximizing the mass of the inner homologous core. The Chandrasekhar mass is smallest for the cooler cores of the lowest-mass supernova progenitors. A sufficiently small, cool, iron core $\left(M_{\text {core }} \sim 1.3 \mathrm{M}_{\odot}\right)$ results from the $11 \mathrm{M}_{\odot}$ progenitor star model of (Woosley \& Weaver 1995) employed in the present work.

A large inner homologous core can result if the lepton fraction is large (Takahara \& Sato 1984, Yamada \& Sato 1994). This can happen if the electron capture rates are slower than the usually employed rates of Fuller, Fowler, \& Newman (1980;1982ab;1985) [hereafter FFN]. Electron capture rates are important because degenerate electrons account for a large fraction of the pressure support of the inner core. A larger $Y_{e}$ therefore implies more pressure support, and a larger inner core. Ultimately, during the collapse, the neutrinos become trapped. The electron capture rates are then suppressed by the high neutrino Fermi energy which limits the available phase space. Afterward, the lepton fraction remains fixed.

The collapse of the inner core slows as the central density exceeds nuclear density and an outward moving shock is produced. A somewhat soft equation of state helps the explosion (Baron \& Cooperstein 1990), but it is not as important as keeping the lepton fraction large.

If the outer part of the core is small enough, it will not completely absorb the outward going shock by the photodissociation of iron-group nuclei. The shock is then not dissipated. It eventually reaches the surface of the outer iron core and an explosion can ensue. Therefore, this combination of conditions (i.e. a large lepton fraction and a small total iron core mass) enables energetic, prompt explosions and the ejection of neutronized material from deep inside the star.

If the standard FFN electron capture rates are employed even smaller iron cores $\left(M \approx 1.2 \mathrm{M}_{\odot}\right)$ cannot explode promptly (Rampp \& Janka 2000, Liebendörfer et al. 2001; cf. Baron \& Cooperstein 1990). With these rates, the lepton fraction is too small and so is the inner core. However, with slower electron capture rates, a prompt explosion is possible. The determination of electron capture rates in supernova cores has, however, suffered from large uncertainties in the associated nuclear physics. Moreover, recent studies (e.g. Langanke \& MartinezPinedo 2000) of electron capture on neutron-rich nuclei have reported rates which are on average an order of magnitude smaller than the previous standard rates of FFN. Indeed, a recent study (Heger et al. 2001) of the effect of these rates on $15-40 \mathrm{M}_{\odot}$ progenitor 
stars found significantly larger $Y_{e}$ and cooler, smaller, cores. Electron capture by free protons in a collapsing core can also be suppressed if the free proton fraction is small. A small proton fraction can result from a large symmetry energy in the equation of state. This is the case for the one we adopt in the current study (Shen et al. 1998a, Shen et al. 1998b).

Clearly, further numerical simulations of the formation and collapse of the small iron cores associated with improved electron capture rates and a good equation of state are called for. This, however, is beyond the scope of the present work. Nevertheless, the model described here is a plausible version of the required physics in order to study the $r$-process elements produced.

\section{Explosion Model}

In the current study, we focus on the consequences for r-process nucleosynthesis of a prompt supernova explosion. In order to investigate mass ejection in a prompt explosion an adiabatic hydrodynamic collapse calculation was performed. As an extreme case, we assume that the electron captures are sufficiently slower than the standard FFN rates that the electron fraction remains fixed during the collapse. This maximizes the shock energy, and therefore, the explosion energy, and brings the iron core to a prompt explosion. We use this hydrodynamical calculation to evaluate the amount of neutron-rich material ejected and the thermodynamic conditions during nucleosynthesis.

Our ultimate goal is a full calculation of the neutrinoradiation hydrodynamics. It is, however, computationally difficult to follow this evolution through the long time scale over which the nucleosynthesis occurs. The work described below is a necessary first preliminary step toward that goal.

\subsection{Equation of State}

One important ingredient for a calculation of a prompt explosion is the nuclear equation of state. Fortunately, recent information on the physics of unstable nuclei is helping to clarify both supernova explosions and the r-process. One can, for example, now use radioactive nuclear beam facilities to probe both the neutron-rich matter of supernovae and the nuclear data needed for r-process nucleosynthesis calculations. Having this experimental information on unstable nuclei with large asymmetry, Sumiyoshi et al. $(1993 ; 1995 \mathrm{a} ; 1995 \mathrm{~b})$ and Hirata et al. (1997) have extensively studied nuclear structure and nuclear matter within a relativistic many-body framework. Based upon this work a relativistic EOS table for application to supernova simulations (Shen et al. 1998a, Shen et al. 1998b) has recently been developed. This relativistic EOS table enables one to perform full simulations of supernovae from the initial gravitational core collapse to the ejection and cooling of material from the newly formed neutron star. It has successfully been applied to studies of neutrinodriven winds from proto-neutron stars Sumiyoshi et al. 2000) and numerical simulations of core collapse (Sumiyoshi et al. 2001). Although this equation of state is not particularly soft (incompressibility $=281$ $\mathrm{MeV})$, it does, have a large symmetry energy (36.9 $\mathrm{MeV})$. Since nuclei with large asymmetries were employed in the development of this equation of state, one expects that the symmetry energy is better determined. The large deduced value reduces the free proton fraction. This can raise the $Y_{e}$ for the core since there are fewer protons available for electron capture.

\subsection{Hydrodynamics}

The prompt supernova calculations described herein are based upon the general-relativistic sphericallysymmetric hydrodynamics code of Yamada (1997). It makes use of a fully implicit Lagrangian numerical scheme. It has been designed for the study of supernova explosions and treats both the hydrodynamics and the neutrino transport.

Although a Boltzmann solver for the neutrino transport has recently been implemented (Yamada, Janka, \& Suzuki 1999) and detailed simulations of gravitational core collapse are now being undertaken, in the current study we have performed calculations of core collapse using only pure hydrodynamics without neutrino transport. Since we are primarily interested here in the dynamics of collapse and prompt ejection, we can as a first approximation ignore the late time neutrino diffusion and heating which is not manifest till later times in the explosion. Indeed, the present work is mostly concerned with matter ejected within $\sim 0.2 \mathrm{sec}$ after core bounce and largely before the arrival of the delayed neutrinos. Obviously, this is an approximation which we will address in a subsequent work.

The present study, however, should be adequate to analyze the physics of the hydrodynamics of core 
collapse, bounce and a possible explosion with our adopted EOS. In particular, our primary purpose here is to evaluate the amount of ejected material which contributes to the r-process. For this particular purpose, our neglect of neutrino radiative transport is probably adequate.

For the same reasons, we assume that the collapse is adiabatic collapse. That is we neglect the heating and cooling via neutrinos. Thus, the entropy per baryon of each mass element remains constant during the collapse except for during the passage of the shock.

Altogether, these approximations tend to maximize the energy of the prompt explosion and provide an upper bound on the amount of ejecta. Thus, our estimates of ejected $r$-process material can perhaps be taken as an upper limit which is what we seek in the present study.

\subsection{Presupernova Model}

We adopt the presupernova model of a $11 M_{\odot}$ progenitor as computed by Woosley \& Weaver (1995). This is the best case for a prompt explosion as it provides the smallest iron core $\left(M_{\text {core }}=1.32 \mathrm{M}_{\odot}\right)$. It is also the smallest progenitor mass among their models ranging from $11 M_{\odot}$ to $40 M_{\odot}$. Both of these conditions are favorable for a prompt explosion. For our hydrodynamic simulations we are only concerned with the central $1.62 M_{\odot}$, which contains the iron core and outer layers. We adopt densities, electron fractions, temperatures and radii as a function of the baryon-mass mesh from the progenitor model. Other quantities are derived using our adopted relativistic EOS table.

The simulations utilize 200 baryon-mass mesh points. This provides enough resolution to analyze the $r$ process in the ejecta. The inner 50 grid points span a range of baryon mass from 0 up to $1.0 M_{\odot}$. The outer 150 grid points span the remaining $0.62 M_{\odot}$. The grid size is taken to be uniform in each of these two regions. We have checked our results in simulations using different mass meshes and find that they do not appreciably alter the nucleosynthesis results.

For the outer boundary conditions, we have fixed quantities at the values obtained at the cut position in the $11 \mathrm{M}_{\odot}$ progenitor model.

\section{Results}


$\sim 0.1-1$, so that heating by the $r$-process was much more important for the dynamics of the ejecta.

\subsection{Electron Fraction in the Ejecta}

During the core collapse, we assume complete suppression of electron capture on neutron-rich nuclei and free protons in the supernova core. We fix $Y_{e}$ to the original values of the progenitor core during the hydrodynamical calculation of the core collapse. (We calculate, however, compositional change after the shock passage separately, to be discussed below.) This assumption overestimates the electron fraction (or lepton fraction after the neutrino trapping) during the collapse. It provides us with a large inner core, and hence, enough shock energy for a successful prompt explosion. We expect that this approximation might lead to an overestimate of the amount of ejected $r$-process material. If that is the case, then it is adequate for our purpose, which is to demonstrate that not too much $r$-process material is ejected.

After the core bounce, the shock passes through the outer part of the iron core and dissociates irongroup nuclei into free neutrons and protons. Near to the remnant, the density reaches $\sim 10^{11} \mathrm{~g} \mathrm{~cm}^{-3}$. Here, electron capture on free protons occurs abruptly because of the high ambient electron degeneracy. The material becomes immediately transparent to neutrinos due to the dissociation of the nuclei which had been the cause of neutrino trapping. Neutrino absorption, which is the reverse of the electron capture, is negligible. This process does not depend upon the details of neutrino transport. It corresponds to the well known neutronization burst in the flux of supernova neutrinos. It causes the material to quickly neutronize right after the shock passes. The resulting neutron-rich region can be generally seen in many supernova simulations (e.g. Suzuki 1994). The inner regions near the nascent neutron star tend to be neutron-rich. It is this electron capture process in the ejecta that produces the neutron excess necessary to achieve r-process conditions.

To treat this neutronization, we have calculated the time evolution of the electron fraction $Y_{e}$ due to electron capture on free protons by postprocessing the results of the hydrodynamical calculation. This electron capture drastically reduces the electron fraction and then ceases quickly due to the rapid expansion of the ejecta. The degree of neutronization depends on the compression of matter at the bounce. The general tendency is that material deeper in the ejecta has a higher electron degeneracy and a smaller electron fraction. The resulting electron fractions in various ejected mass shells then become initial conditions for our subsequent $\mathrm{r}$-process nucleosynthesis calculations (see Figure 2d).

We should point out that this explicit determination of the neutronization of the ejecta is an improvement over the previous hydrodynamic calculations of Hillebrandt et al. (1976). In that work a continuous distribution in $Y_{e}$ between $Y_{e}=0.5$ at the base of the silicon-burning shell and $Y_{e}=0.125$ at the remnant neutron star was simply assumed for the $r$-process initial conditions. As we shall see, our calculations indicate that a much smaller region of neutronized material actually emerges from a prompt explosion.

Figure 3 summarizes the electron fraction of material at the beginning of nucleosynthesis as a function of baryon mass coordinate in the ejecta. The dotted line displays the initial value of $Y_{e}$ before the neutronization. As we shall see the $r$-process occurs only in regions with $Y_{e} \sim 0.15$ to 0.25 . In this calculation that corresponds to only $0.025 \mathrm{M}_{\odot}$ of material. This is much less than in the earlier estimates of Hillebrandt et al (1976). The difference between the present results and their calculation can be traced to their simplified treatment of the neutronization of the ejecta. We stress again that we have performed hydrodynamical calculations to find the ejected part of material and have explicitly calculated the electron fractions of each mass elements due to electron captures on free protons in order to determine both the amount and neutron-richness of ejecta.

The grid point $90\left(M_{b}=1.163 M_{\odot}\right.$ at zone center in baryon mass coordinate) is the inner-most mass shell of the ejected material. This trajectory has the smallest electron fraction $\left(Y_{e}=0.16\right.$ in Figure 3$)$. It contributes the most to the $r$-process elements around the $\mathrm{A}=195$ abundance peak. The grid points from 90 to $95\left(M_{b}=1.184 M_{\odot}\right)$, leading to $Y_{e} \lesssim 0.25$ contribute to the r-process nucleosynthesis from $\mathrm{A}=130$ to $\mathrm{A}=190$. The grid point $111\left(M_{b}=1.250 M_{\odot}\right)$ has an electron fraction 0.45 . This trajectory only produces elements up to $A=70$ and does not contribute to the $r$-process. Accordingly, we do not add outer mass shells (grid points $>112$ ) in the following nucleosynthesis calculations as they do not contribute to the abundances of $r$-process elements. 


\section{5. r-Process Nucleosynthesis}

As the neutronized material is ejected it quickiy experiences charged-particle and neutron-capture reactions to produce seed material followed by neutron captures and beta decays along the $r$-process path. The $r$-process nucleosynthesis calculations in the present work are based upon the nuclear reaction network as described in Terasawa et al. (2001). In that work it was shown that both neutron-rich lightmass nuclei as well as heavy nuclei can play an important role in the production of both seed nuclei and $r$-process elements. The path through light nuclei is most important in very neutron rich conditions such as in the high entropy neutrino-driven wind or in the most neutron-rich ejecta studied here. We thus make use of an extended nuclear reaction network which includes all relevant nuclear reactions from protons to heavy actinides (Terasawa et al. 2001). The nuclear reaction network includes nuclei from beta-stability to the neutron-drip line. For $10 \leq Z \leq 94$, we have used the network of Meyer et al. (1992), which includes about 3000 nuclear species. We have extended this network to include lighter nuclei as well as almost all charged-particle reactions for $\mathrm{A} \leq 28$ Kajing $\&$ Boyd 1990: Orito et al. 1997) plus all $(\alpha, n)$ reactions up to $\mathrm{Z}=36$. For the nucleosynthesis calculation we have checked the results with and without $\nu_{e}$ capture on nuclei (Meyer, McLaughlin, \& Fuller 1998). The results do not depend upon this effect, presumably because material is so quickly removed from the neutron star surface.

\section{1. $\quad r$-Process Yields}

The ejected mass shells of Figure 1 were evolved with our $r$-process network. Figure 4 shows the results of several trajectories labeled by their Lagrangian zone numbers. We see that material ejected at the bottom contribute to the heaviest $A=195$ peak, while those shells which had higher $Y_{e}$ only contribute to the $A=130$ peak and below. Shells with $Y_{e}>0.25$ only produce iron group and elements up to $A=100$. They do not contribute to the synthesis of $r$-process elements.

The nucleosynthesis yields of these different mass shells were summed to produce the final $r$-process abundance curve shown in Figure 5. Here, we see an adequate reproduction of the Solar $r$-process abundances. The dips above and below the $A \approx 130$ peak in our computed abundance curve may be an indication (cf. Meyer et al. 1992) that the $N=82$ shell closure is too strong in our mass formula Hilf et al. 1976). Other than that, the abundances of nuclei heavier than $A \sim 100$ are nicely reproduced.

The fact that elements with $A<100$ are underproduced suggests an interesting possibility. These are precisely the nuclei overproduced in the neutrinodriven wind scenario. They are also the elements which seem to deviate from the Solar $r$-process abundances in metal-poor halo stars (Ryan, Norris, \& Beers 1996; Sneden et al. 1996; 1998; 2000). The suggestion is therefore that two different environments may contribute to the $r$-process: one being the prompt explosion of low-mass SNe progenitors under study here. Such environments may produce the heaviest $r$-process nuclei; the other environment could be the neutrino-driven winds in more massive progenitors. These events might produce the lighter $A \leq 100$ nuclides.

This is consistent with recent meteoritic evidence (Qian 2000; Qian \& Wasserburg 2000) that two different $r$-process environments preceded the formation of the Solar System. It is also consistent with galactic evolution evidence. Ishimaru \& Wanajo (1999) have demonstrated that the observed large dispersion of $r$-process elements in metal-poor halo stars can be explained if the r-process occurred in either low-mass $\left(8-10 \mathrm{M}_{\odot}\right)$ or high mass $\left(\sim 30 \mathrm{M}_{\odot}\right)$ supernova progenitors. Here we favor low-mass progenitors, but perhaps both environments contribute.

\subsection{Galactic Contribution}

Regarding the $r$-process yields implied by the present study, we see from Figure 3 that $\approx 0.025 \mathrm{M}_{\odot}$ of $r$-process material is ejected in our $11 \mathrm{M}_{\odot}$ model. This corresponds to a mass fraction in the ejecta of $X_{r}(m=11) \approx 3 \times 10^{-3}$. However, since the prompt mechanism can only work in low mass progenitors, only supernovae in a narrow mass range, say $\Delta m \sim 1$ $\mathrm{M}_{\odot}$ probably contribute to the galactic abundances. Hence, to estimate the average yield per supernova, we must correct for the fraction of supernovae which can contribute. Assuming a standard Salpeter initial mass function $\phi(m) \propto m^{-2.3}$, then the total average mass fraction of ejected $r$-process material per supernova $\left\langle X_{r}\right\rangle_{S N}$ becomes:

$$
\left\langle X_{r}\right\rangle_{S N}=\frac{X_{r}(m=11) \times\left(m-m_{r e m}\right) \phi(11) \Delta m}{\int_{11}^{\infty}\left(m-m_{\text {rem }}\right) \phi(m) d m}
$$




$$
\approx \frac{0.3 X_{r}(m=11) \Delta m}{11 \mathrm{M}_{\odot}} \approx 7.5 \times 10^{-5}
$$

where in the above we have ignored the mass of the remnant compared to the mass of ejecta. Now assuming that the total SNII rate has been constant at $10^{-2}$ $\mathrm{yr}^{-1}$ over the past $10^{10} \mathrm{yr}$, then we would estimate a current mass in $r$-process elements of the Galaxy of $M_{r}^{\text {Gal }} \approx 7.5 \times 10^{4} \mathrm{M}_{\odot}$. Assuming a total baryonic mass for the Galaxy of $10^{11} \mathrm{M}_{\odot}$, then the present mass fraction in $r$ elements would be $\sim 7.5 \times 10^{-7}$ in reasonable agreement with the observed Solar mass fraction of $\sim 10^{-7}$.

\section{Conclusion}

We conclude that the prompt mechanism in lowmass supernova progenitors remains as a viable model for the $r$-process. There is no over-production of $r$ process elements as long as an accurate accounting of the neutronization of the prompt ejecta is made. It also provides a very good reproduction of the Solar $r$-process abundance distribution for elements with $A \geq 100$, suggesting that this might be the source of the heaviest $r$-process nuclei while perhaps delayed neutrino-driven winds or neutron-star mergers may be the source of lighter $r$-process nuclei. Obviously, a key component of this paradigm is to understand just which progenitors are capable of producing a prompt explosion and $r$-process nucleosynthesis and also whether the late time neutrino heating will affect the $r$-process yields. In future work we will address both of these points, however, regarding the delayed neutrino emission we expect that it will not affect the results reported here as by the time the delayed neutrinos arrive, the prompt ejecta is already far from the proto-neutron star.

The authors wish to thank J. Cowan, W. Hillebrandt and S. Wanajo for useful discussions on the $r$-process. We also thank Stan Woosley for providing his numerical data of presupernova models. One of the authors (KS) would like to acknowledge the collaborative efforts of H. Shen, K. Oyamatsu and H. Toki for providing the relativistic EOS table. Numerical simulations have been performed on the supercomputers at RIKEN and KEK (KEK Supercomputer Project No. 00-63, 01-75). One of the authors (MT) wishes to acknowledge fellowship support from RIKEN as a Junior Research Associate. This work was supported in part by Japan Society for Promotion of Science, and by the Grant-in Aid for Scientific Research (1064236, 10044103, 11127220, 12047230, 12740138) of the Ministry of Education, Science, Sports and Culture of Japan. Work at Univ. Notre Dame is supported by DoE Nuclear Theory Grant DE-FG02-95-ER40934.

\section{REFERENCES}

Baron, E. \& Cooperstein, J. 1990, ApJ, 353, 597

Bethe, H. 1990, Rev. Mod. Phys, 62, 801

Fuller, G. M., Fowler, W. A., \& Newman, M. J. 1980, ApJS, 42, 447

Fuller, G. M., Fowler, W. A., \& Newman, M. J. 1982a, ApJ, 252, 715

Fuller, G. M., Fowler, W. A., \& Newman, M. J. 1982b, ApJS, 48, 279

Fuller, G. M., Fowler, W. A., \& Newman, M. J. 1985, ApJ, 293, 1

Freiburghaus, C., Rosswog, S., \& Thielemann, F. -K. 1999, ApJ, 525, L121

Hilf, E. R., von Groote, H., \& Takahashi, K. 1976, in Proc. 3rd Int. Conf. on Nuclei far from Stability (Geneva: CERN) 76-13, p. 142.

Heger, A., Langanke, K., Martinez-Pinedo, G., \& Woolsey, S.E. 2001, Phys. Rev. Lett., 86, 1678

Hillebrandt, W. 1978, Sp. Sci. Rev., 21, 639

Hillebrandt, W. 1982, A\&A, 110, L3

Hillebrandt, W., Nomoto, K., \& Wolff, R. G. 1984, A\&A, 133, 175

Hillebrandt, W., Takahashi, K., \& Kodama, T. 1976, A\&A, 52, 63

Hirata, D. Sumiyoshi, K. Tanihata, I. Sugahara, Y. Tachibana, T., \& Toki, H. 1997, Nucl. Phys. A, $616,438 \mathrm{c}$

Ishimaru, Y. \& Wanajo, S. 1999, ApJ, 511, L33

Kajino, T. \& Boyd, R. N. 1990, ApJ, 359, 267

Käppeler, F., Beer, H., \& Wisshak, K. 1989, Rep. Prog. Phys., 52, 945 
Langanke, K. \& Martinez-Pinedo G. 2000, Nucl. Phys. A673, 481

Liebendörfer, M., Mezzacappa, A., Thielemann, F.K., Messer, O.E.B., Hix, W.R. \& Bruenn, S.W. 2001, Phys. Rev. D in press, astro-ph/0006418

Mathews, G. J. \& Cowan, J. J. 1990, Nature, 345, 491

Mathews, G. J. \& Ward, R. A. 1985, Rep. Prog. Phys., 48, 1371

Meyer, B. S. 1994, ARA\&A, 32, 153

Meyer, B. S., Mathews, G. J., Howard, W. M., Woosley, S. E., \& Hoffman, R. D. 1992, ApJ, 399, 656

Meyer, B. S., McLaughlin, G. C., \& Fuller G. M. 1998, Phys. Rev. C, 58, 3696

Orito, M., Kajino, T., Boyd, R. N., \& Mathews, G. J. 1997, ApJ, 488, 515

Otsuki, K., Tagoshi, H., Kajino, T., \& Wanajo, S. 2000, ApJ, 533, 424

Qian, Y. -Z. 2000, ApJ, 534, L67

Qian, Y. -Z. \& Wasserburg, G. J. 2000, Phys. Rep., 333,77

Qian, Y. -Z. \& Woosley, S. E. 1996, ApJ, 471, 331

Rampp, M. \& Janka, H.-T. 2000, ApJ, 539, L33

Ryan, S. G., Norris, J. E., \& Beers, T. C. 1996, ApJ, 471,254

Sato, K. 1974, Prog. Theor. Phys., 51, 726

Shen, H., Toki, H., Oyamatsu, K., \& Sumiyoshi, K. 1998a, Nucl. Phys. A, 637, 435

Shen, H., Toki, H., Oyamatsu, K., \& Sumiyoshi, K. 1998b Prog. Theor. Phys., 100, 1013

Sneden, C., Cowan, J. J., Debra, L. B., \& Truran, J. W. 1998, ApJ, 496, 235

Sneden, C., Cowan, J. J., Ivans, I. I. Fuller, G. M. Burles, S., Beers, T. C., \& Lawler, J. E. 2000, ApJ, 533, L139

Sneden, C., McWilliam, A., Preston, G. W., Cowan, J. J., Burris, D. L., \& Armosky, B. J. 1996, ApJ, 467,819
Sumiyoshi, K., Hirata, D., Toki H., \& Sagawa, H. 1993, Nucl. Phys., A552, 437

Sumiyoshi, K., Kuwabara H., \& Toki, H. 1995a, Nucl. Phys. A, 581, 725

Sumiyoshi, K., Suzuki, H., Otsuki, K., Terasawa, M., \& Yamada, S. 2000, PASJ, 52, 601

Sumiyoshi, K. Suzuki H., \& Toki, H. 1995b, A\&A, 303,475

Sumiyoshi, K., Suzuki, H., Yamada S., \& Toki, H. 2001, in preparation for submission to Nucl. Phys.

Suzuki, H. 1994, in Physics and Astrophysics of Neutrinos, edited by Fukugita, M. and Suzuki, A., (Springer-Verlag, Tokyo, 1994), p.780 and references therein.

Takahara, M. \& Sato, K. 1984, Prog. Theor. Phys. 71,524

Takahashi, K., Witti, J., \& Janka, H. -Th. 1994, A\&A, 286, 857

Terasawa, M. Sumiyoshi, K. Kajino, T. Mathews, G. J., \& Tanihata, I. 2001, submitted to ApJ.

Wheeler, J. C., Cowan, J. J., \& Hillebrandt, W. 1998, ApJ, 493, L101

Witti, J., Janka, H. -Th., \& Takahashi, K. 1994, A\&A, 286, 841

Woosley, S. E. \& Hoffman, R. D. 1992, ApJ, 395, 202

Woosley, S. E., Wilson, J. R., Mathews, G. J., Hoffman, R. D., \& Meyer, B. S. 1994, ApJ, 433, 229

Woosley, S.E. \& Weaver, T.A. 1995, ApJS, 101, 181

Yamada, S. 1997, ApJ, 475, 720

Yamada, S. \& Sato, K. 1994, ApJ, 434, 268

Yamada, S. Janka, H.-Th., \& Suzuki, H. 1999, A\&A, 344,533

This 2-column preprint was prepared with the AAS LATEX macros $\mathrm{v} 4.0$. 


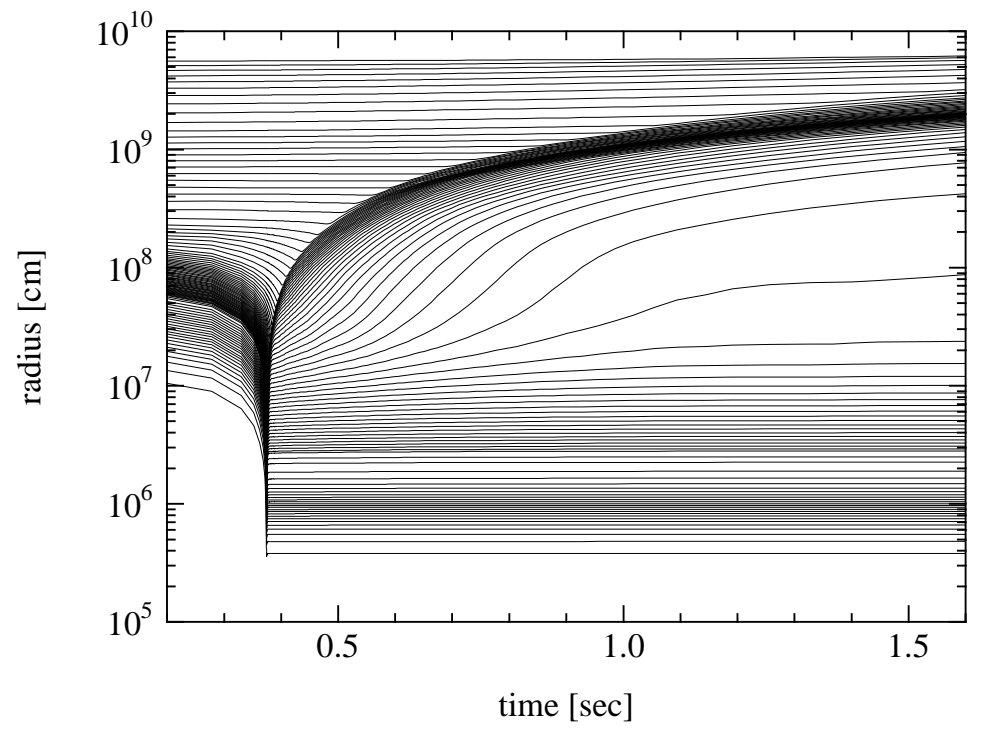

Fig. 1.- Evolution of the spatial coordinate for various mass shells in the collapse and prompt explosion of our 11 $\mathrm{M}_{\odot}$ model. 

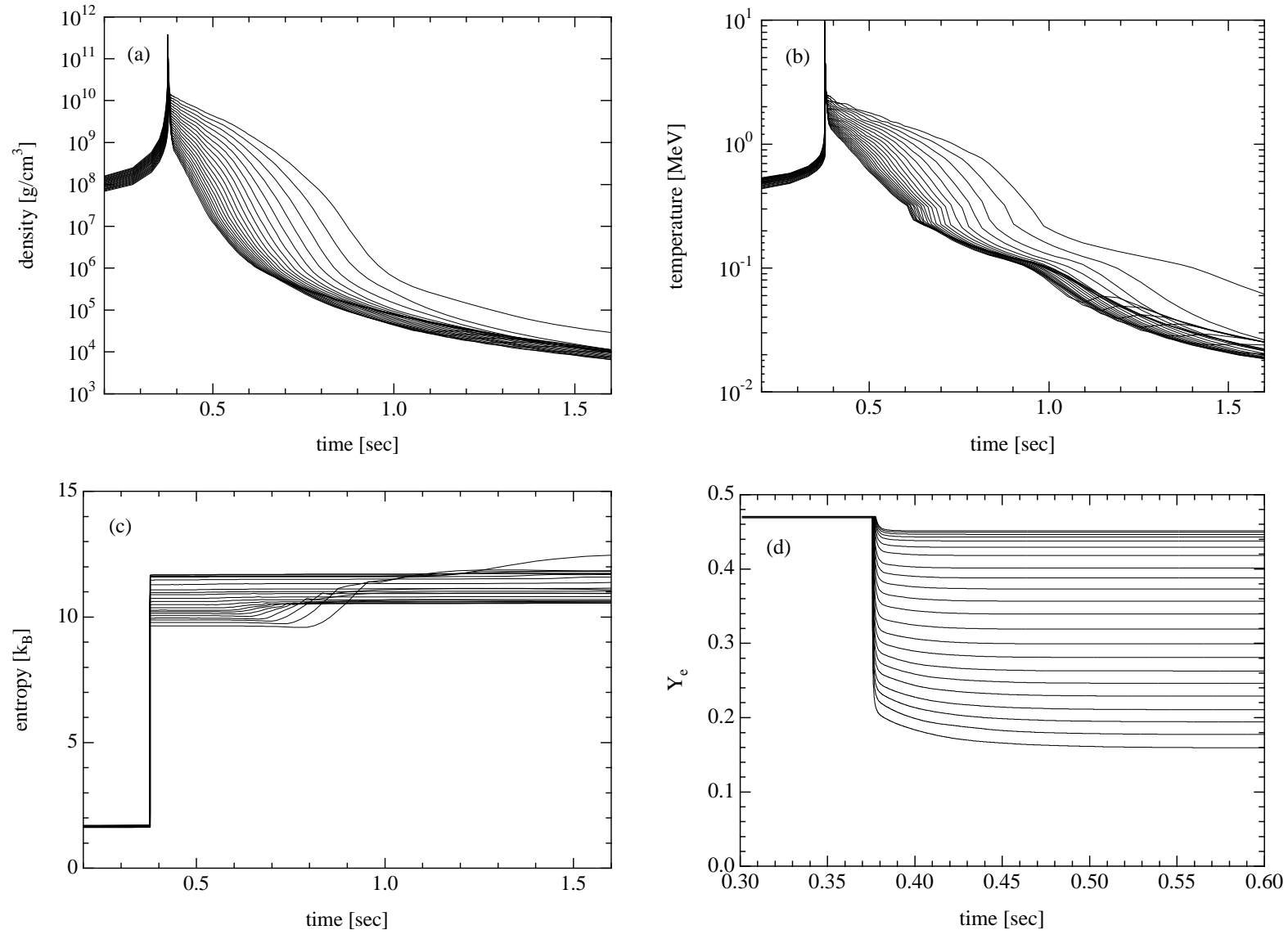

Fig. 2.- Evolution of: (a) baryonic mass density; (b) temperature; (c) entropy; and (d) electron fraction $Y_{e}$ as a function of time for ejected material in the prompt explosion. Note that the time interval is expanded for (d) to better show the rapid change in $Y_{e}$. 


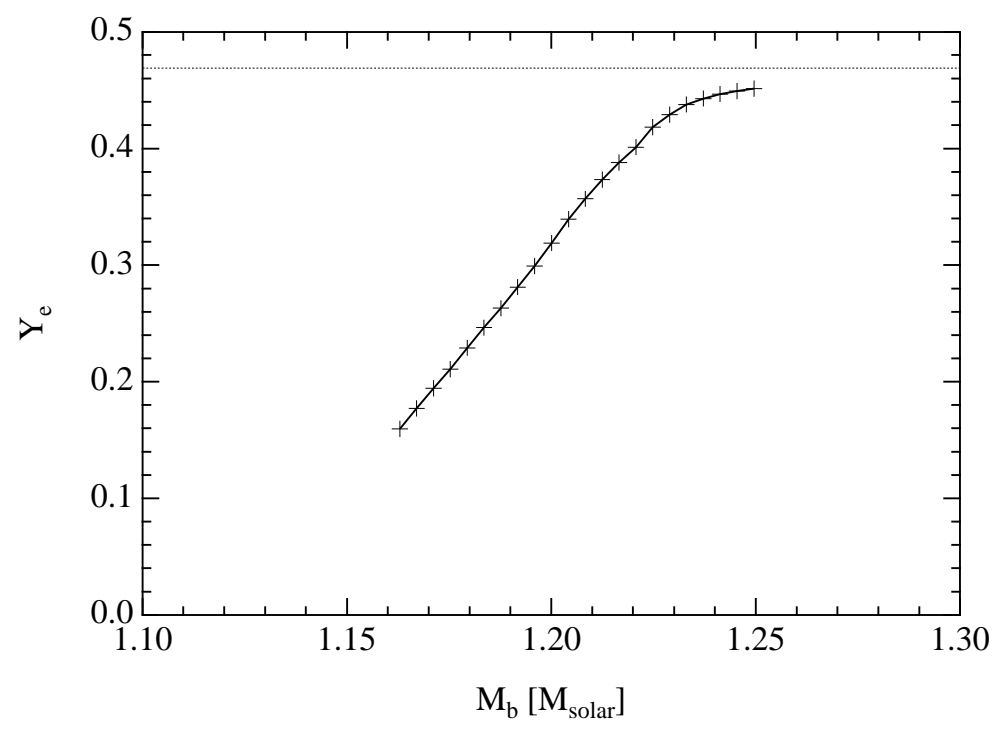

Fig. 3.- Electron fraction $Y_{e}$ as a function of baryon mass coordinate for ejected material in the prompt explosion.

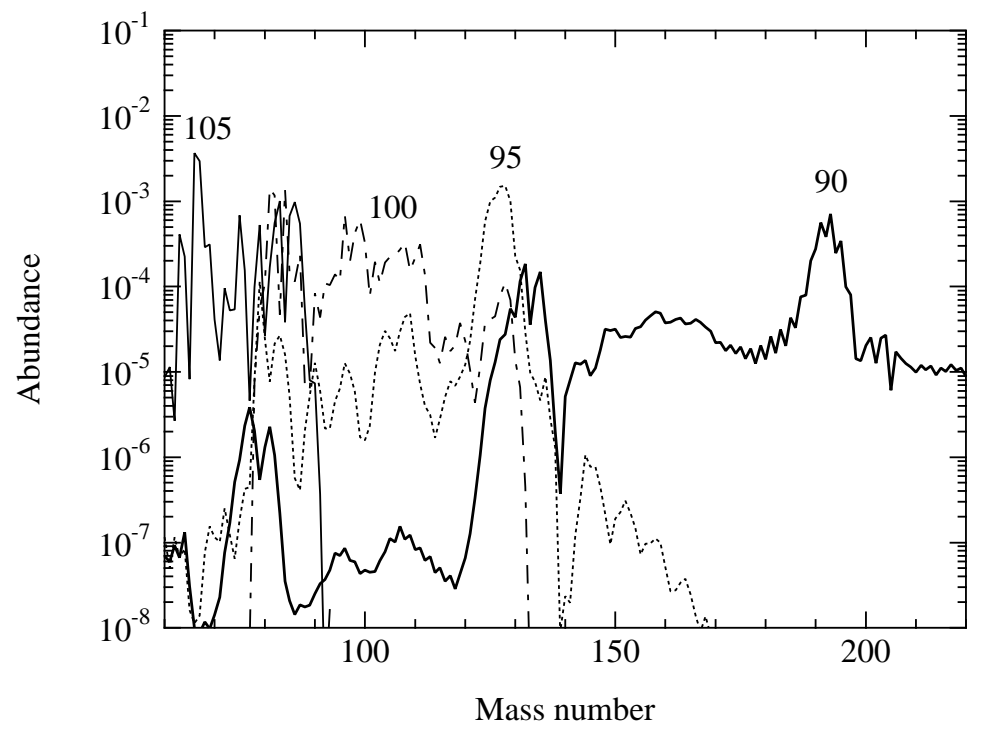

Fig. 4.- Abundances computed for ejected material from several representative mass shells: $90\left(Y_{e}=0.16\right) ; 95$ $\left(Y_{e}=0.25\right) ; 100\left(Y_{e}=0.34\right) ; 105\left(Y_{e}=0.42\right)$. 


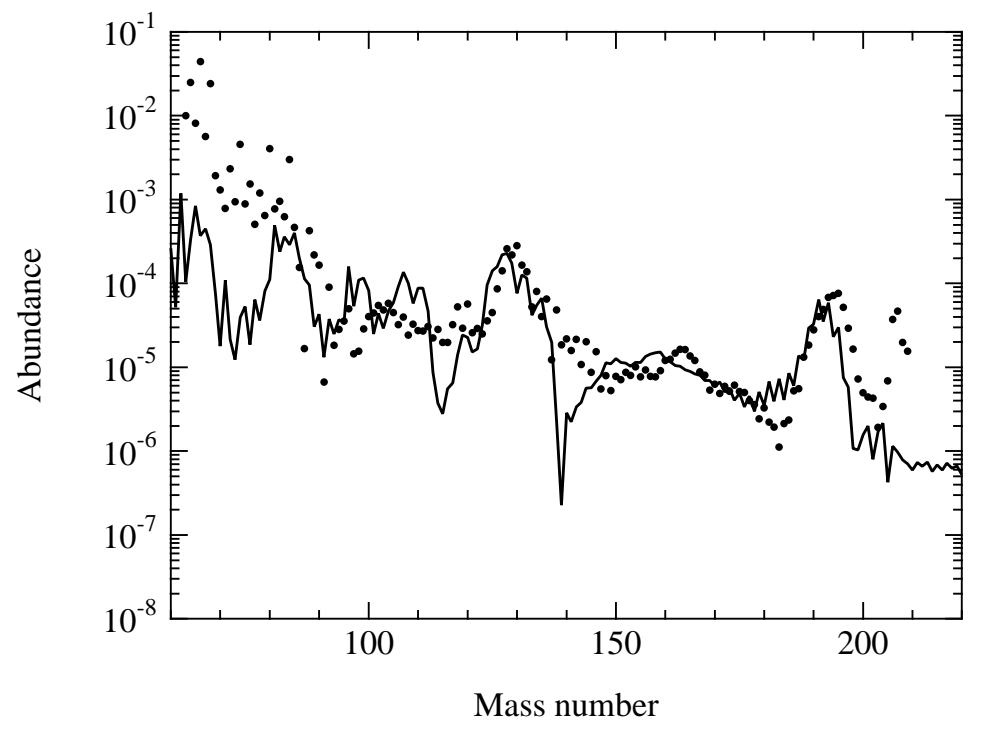

Fig. 5.- Final integrated isotopic abundances for ejected material (solid line) compared with the Solar $r$-process abundances (filled circles; Käppeler, Beer, \& Wisshak 1989). 